\title{
CFD solver validations for simulating passively pitching tandem wings in hovering flight
}

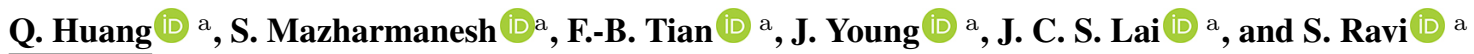 \\ ${ }^{a}$ University of New South Wales at Canberra, GPO BOX 7916, Canberra ACT 2610, Australia \\ Email: qxhuang2013@gmail.com
}

\begin{abstract}
Wing pitching motion in insect flapping flight has been recognized as a passive phenomenon induced by inertial and aerodynamic forces. Inspired by the insect flight, passively pitching flapping wings have been implemented in micro aerial vehicles (MAVs) designs. The pitching angle of the flapping wings is passively modulated by an elastic hinge using a torsional spring. In order to understand the complex passive pitching mechanisms, various experimental and numerical efforts have been made. However, the passive pitching mechanisms in tandem ipsilateral wings (e.g. dragonfly) remain unclear as the wing-wing interactions are complex. Here, passive pitching of tandem rectangular wings in free hovering condition is numerically simulated using an immersed boundary-lattice Boltzmann method (IB-LBM). Validation of the solver was performed by simulating rectangular flapping plate within prescribed kinematics and a rigid fruit fly wing with passive pitching. Good agreement of results between current computations and published data were observed, suggesting that the present computational fluid dynamics (CFD) solver can accurately compute passively pitching flapping wing systems. The high-fidelity and efficiency were also investigated by performing grid convergence studies and comparing the computational time with previously published data. This study provides additional data for benchmarking of CFD solvers in the simulation of passive flapping wings. The benchmark is also extended by simulating passive pitching of tandem dragonfly wings in hovering flight.
\end{abstract}

Keywords: Passive pitching, fluid-structure interaction, tandem flapping wings, insect flight, dragonfly 


\section{INTRODUCTION}

Many insects have spring-like elements in the form of elastic materials in their thoraxes, muscles, and tendons that may reduce the energetic demands of flapping flight and improve flight efficiency (Lynch et al. 2021). During the flapping flight, insects apply their muscle forces through the axis close to the leading edge, which creates a moment with respect to the mass centre of the wing, leading to the passive pitching of the wing due to the interaction between the wing and the surrounding airflow (Ennos 1988). Inspired by insect flight, passively pitching flapping wings have been implemented in micro aerial vehicles (MAVs) designs Farrell Helbling \& Wood (2018). In order to understand the passive pitching mechanism of flapping wings, various experimental and numerical studies have been conducted (Mazharmanesh et al. 2021, Lei \& Li 2020). However, passive pitching of tandem ipsilateral wings (e.g. dragonfly) has not been fully explored. Here, the computation of a rigid rectangular flapping plate with prescribed flapping and pitching motions and a rigid fruit fly wing with passive pitching in hovering flight are conducted to validate the present immersed boundary-lattice Boltzmann method (IB-LBM) solver. The validated solver is then applied to simulate the passive pitching of tandem rectangular wings.

\section{MODEL DESCRIPTION}

The unsteady incompressible flow is governed by the continuity and Navier-Stokes equations

$$
\nabla \cdot \boldsymbol{u}=0, \quad \frac{\partial \boldsymbol{u}}{\partial t}+\boldsymbol{u} \cdot \nabla \boldsymbol{u}=-\frac{1}{\rho} \nabla p+\nu \nabla^{2} \boldsymbol{u}+\boldsymbol{f}
$$

where $\boldsymbol{u}$ is the fluid velocity, $\rho$ is the constant density, $\boldsymbol{f}$ is the body force and $p$ and $\nu$ are pressure and kinematic viscosity, respectively.

\section{NUMERICAL METHOD}

The D3Q19 lattice Boltzmann method (LBM) with multi-relaxation-time (MRT) model is adopted for simulation of the fluid dynamics. The two-way fluid-structure interactions are coupled by a feedback immersed boundary method (IBM). In the LBM, the macroscopic dynamics of the fluid are the result of the statistical behaviour of the particles, which are described by the distribution function $g_{i}(\boldsymbol{x}, t)$. The evolution of this distribution function is according to

$$
g_{i}\left(\boldsymbol{x}+\boldsymbol{e}_{i} \Delta t, t+\Delta t\right)-g_{i}(\boldsymbol{x}, t)=\Omega_{i}(\boldsymbol{x}, t)+\Delta t G_{i},
$$

where $g_{i}(\boldsymbol{x}, t)$ is the distribution function for particles with velocity $\boldsymbol{e}_{i}$ at position $\boldsymbol{x}$ and time $t, \Delta t$ is the time increment, $\Omega_{i}(\boldsymbol{x}, t)$ is the collision operator and $G_{i}$ is the forcing term accounting for the body force $f$. The D3Q19 model (D'Humieres et al. 2002) is used on a cube lattice. The MRT collision model is adopted and is given by

$$
\Omega_{i}=-\left(\boldsymbol{M}^{-1} \boldsymbol{S} \boldsymbol{M}\right)_{i j}\left[g_{i}(\boldsymbol{x}, t)-g_{i}^{e q}(\boldsymbol{x}, t)\right],
$$

where $\boldsymbol{M}$ is a $19 \times 19$ transform matrix for D3Q19 model and $\boldsymbol{S}=\operatorname{diag}\left(\tau_{0}, \tau_{1}, \ldots, \tau_{18}\right)^{-1}$ is a non-negative diagonal relaxation matrix. The determination of $\boldsymbol{S}$ in three-dimensional model can be found in D'Humieres et al. (2002). The equilibrium distribution function $g_{i}^{e q}$ is defined as

$$
g_{i}^{e q}=\rho \omega_{i}\left[1+\frac{\boldsymbol{e}_{\boldsymbol{i}} \cdot \boldsymbol{u}}{c_{s}^{2}}+\frac{\boldsymbol{u} \boldsymbol{u}:\left(\boldsymbol{e}_{i} \boldsymbol{e}_{i}-c_{s}^{2} \boldsymbol{I}\right)}{2 c_{s}^{4}}\right],
$$

where $c_{s}=\Delta x /(\sqrt{3} \Delta t)$ is the speed of sound, $\Delta x$ is the lattice spacing, $\boldsymbol{I}$ is the unit tensor and the weighting factors $\omega_{i}$ are given by $\omega_{0}=1 / 3, \omega_{1-6}=1 / 18$ and $\omega_{7-18}=1 / 36$. The velocity $\boldsymbol{u}$, mass density $\rho$ and pressure $p$ can be obtained according to

$$
\rho=\sum_{i} g_{i}, \quad p=\rho c_{s}^{2}, \quad \boldsymbol{u}=\left(\sum_{i} \mathbf{e}_{i} g_{i}+\frac{1}{2} \boldsymbol{f} \Delta t\right) / \rho
$$

The force scheme proposed in Guo et al. (2002) is adopted to determine $G_{i}$,

$$
\begin{aligned}
G_{i} & =\left[\boldsymbol{M}^{-1}(\boldsymbol{I}-\boldsymbol{S} / 2) \boldsymbol{M}\right]_{i j} F_{i}, \\
F_{i} & =\left(1-\frac{1}{2 \tau}\right) \omega_{i}\left[\frac{\boldsymbol{e}_{i}-\boldsymbol{u}}{c_{s}^{2}}+\frac{\boldsymbol{e} \cdot \boldsymbol{u}}{c_{s}^{4}} \boldsymbol{e}_{i}\right] \cdot \boldsymbol{f},
\end{aligned}
$$


Q. Huang et al., CFD solver validations for simulating passively pitching tandem wings in hovering flight

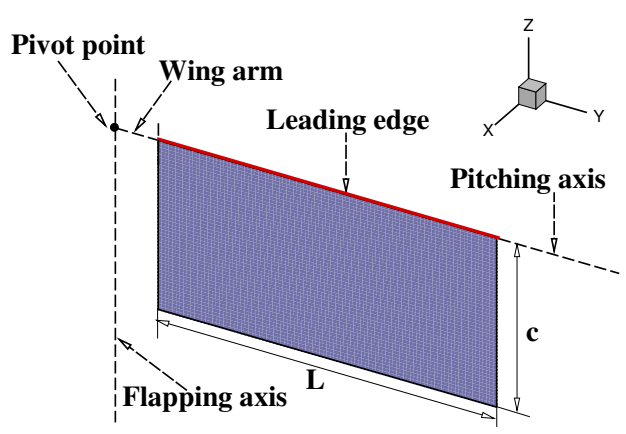

(a) 3D view

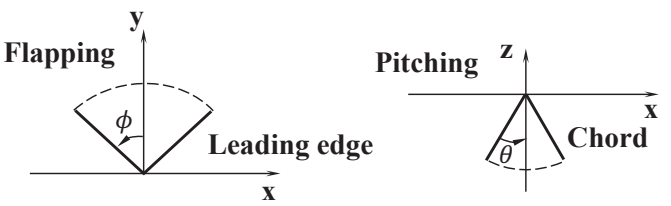

(b) $2 \mathrm{D}$ view

Figure 1. Schematic diagram of the rectangular wing model used in the study.

where $\tau$ is the non-dimensional relaxation time.

In the present study a feedback IBM (Huang et al. 2021a) is adopted to handle the no-slip boundary conditions on the flapping wing. In this method a body force $f$ is added in the Navier-Stokes equation to mimic a boundary condition according to

$$
\begin{aligned}
& \boldsymbol{f}(\boldsymbol{x}, t)=-\int \boldsymbol{F}_{i b}(s, t) \delta(\boldsymbol{x}-\boldsymbol{X}(s, t)) d A, \\
& \boldsymbol{F}_{i b}(s, t)=\alpha \rho(\boldsymbol{x}, t)\left(\boldsymbol{U}_{i b}(s, t)-\boldsymbol{U}(s, t)\right), \\
& \boldsymbol{U}_{i b}(s, t)=\int \boldsymbol{u}(x, t) \delta(\boldsymbol{x}-\boldsymbol{X}(s, t)) d \boldsymbol{x},
\end{aligned}
$$

where $\boldsymbol{F}_{i b}(s, t)$ is the Lagrangian force density, $d A$ is the element surface area of the immersed boundary, $\delta(\boldsymbol{x}-\boldsymbol{X}(s, t))$ is Dirac's delta function, $\boldsymbol{x}$ is the coordinate of the fluid lattice nodes, $\boldsymbol{X}$ is the coordinate of the structure (i.e. the flapping wing here), $\alpha$ is the feedback coefficient and $\alpha=2 s$ in LBM simulations. In dimensionless form $\alpha^{*}=\alpha /\left(U_{\text {ref }} / L_{\text {ref }}\right)=40$, and $\alpha^{*}$ ranges from 20 to 104 (Huang et al. 2021a). Here $U_{r e f}$ and $L_{r e f}$ are the reference velocity and length, respectively. $\boldsymbol{U}_{i b}(s, t)$ is the immersed boundary velocity obtained by interpolation at the immersed boundary and $\boldsymbol{U}(s, t)$ is the velocity of the wing. The 4-point discrete delta function $\delta_{h}(\boldsymbol{x})$ is used to approximate the Dirac delta function Peskin (2002),

$$
\begin{array}{rc}
\delta_{h}(\boldsymbol{x})=\frac{1}{\Delta x \Delta y \Delta z} \zeta\left(\frac{x}{\Delta x}\right) \zeta\left(\frac{y}{\Delta y}\right) \zeta\left(\frac{z}{\Delta z}\right), & \\
\zeta(r)=\frac{1}{8}\left(3-2|r|+\sqrt{1+4|r|-4 r^{2}}\right) & 0 \leq|r| \leq 1, \\
\frac{1}{8}\left(5-2|r|+\sqrt{-7+12|r|-4 r^{2}}\right) & 1 \leq|r| \leq 2, \\
0 & |r|>2 .
\end{array}
$$

\section{VALidations}

The numerical method used here has been extensively validated and applied in confined flows (e.g. 2D collapsible channel flows and 3D collapsible tube flows) in our previous publications (Huang et al. 2020a, 2021a,b). Here the simulations of a rectangular flapping wing and a fruit fly flapping wing are further considered to validate the computations of 3D flapping wings. To reduce the computational effort, a multi-block LBM (Yu et al. 2002) is adopted to provide high resolution near the solid body, with low resolution in the farfield. A hybrid open multi-processing (OpenMP) and message passing interface (OpenMPI) parallel computing strategy has been incorporated into the code to accelerate the computation.

\subsection{A rigid rectangular flapping plate with prescribed flapping and pitching motions}

A thin and rigid rectangular plate in hovering flight with prescribed flapping and pitching motions is considered, as shown in figure 1 . The wing has a chord length $c$ and a span of $L=2 c$. The aspect ratio is 
Q. Huang et al., CFD solver validations for simulating passively pitching tandem wings in hovering flight

Table 1. Fluid domain size in $x-, y-$, and $z-$ directions, finest grid size, grid number, and CPU time $t_{w}$ of one stroke cycle for the computation of the rectangular wing model. $h$ : hour.

\begin{tabular}{lllll}
\hline Sources & Domain size & Finest grid size & Grid number $\left(10^{6}\right)$ & $t_{w}(h) /$ cycle \\
\hline Dai et al. (2012) & {$[14 \mathrm{c}, 15 \mathrm{c}, 15 \mathrm{c}]$} & $0.05 \mathrm{c}$ & 1.72 & 14 \\
Present IB-LBM & {$[14 \mathrm{c}, 15 \mathrm{c}, 15 \mathrm{c}]$} & $0.05 \mathrm{c}$ & 3.14 & 0.18 \\
\hline
\end{tabular}

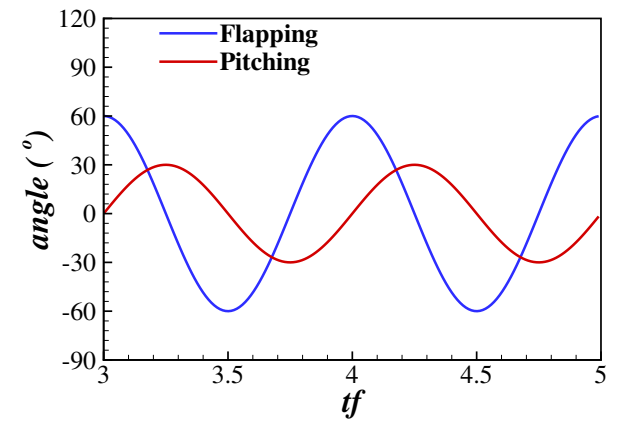

(a) Wing kinematics

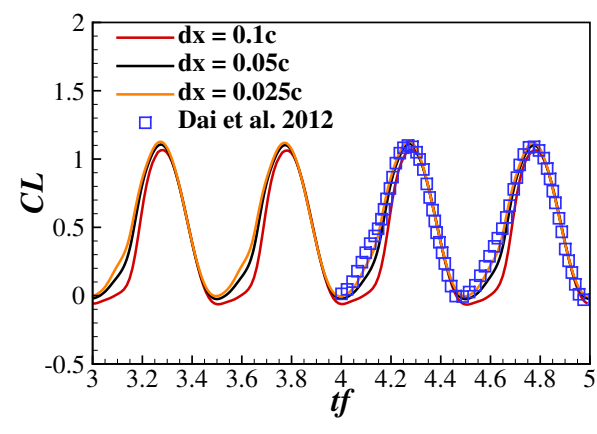

(b) $C_{L}$

Figure 2. Rectangular wing: wing kinematics in two stroke cycles and the time history of the lift coefficient.

$A R=L / c=2.0$. The leading edge undergoes two degrees-of-freedom rotations as the torques activate the wing at the pivot point (Dai et al. 2012),

$$
\phi=\frac{A_{\phi}}{2} \sin \left(2 \pi f t+\frac{\pi}{2}\right), \quad \theta=\frac{A_{\theta}}{2} \sin (2 \pi f t),
$$

where $\phi$ is the flapping angle, $\theta$ is the pitching angle, $f$ is the flapping frequency, and $A_{\phi}=2 \pi / 3$ and $A_{\theta}=\pi / 3$ are the flapping and pitching amplitudes, respectively. The wing arm (from the pivot point to the wing root) has length $0.1 c$. The Reynolds number, drag and lift coefficients are defined as

$$
\operatorname{Re}=\frac{U c}{\nu}=176, \quad C_{D}=\frac{2 F_{x}}{\rho U^{2} A}, \quad C_{L}=\frac{2 F_{z}}{\rho U^{2} A},
$$

where $U$ is the mean wingtip velocity at the leading edge and $U=2 A_{\phi} f(L+0.1 c)=8.797 c f$. Here $F_{x}$ and $F_{z}$ are the force acting on the wing by the ambient fluid in $x$ and $z$ direction, respectively. $A$ is the surface area of the wing ( $A=c L$ for a rectangular plate). As shown in table 1 , the rectangular computational domain has a size of $14 c \times 15 c \times 15 c$, which is the same size as that of Dai et al. (2012). The most refined grid around the wing is $0.05 c$, and the total grid number is $3.14 \times 10^{6}$. The CPU time per one stroke cycle of the present IB-LBM solver is almost two orders of magnitude lower than that in Dai et al. (2012). Five stroke cycles are simulated to ensure that all the force histories (e.g. $C_{D}$ and $C_{L}$ ) have reached steady state. Dirichlet boundary conditions for the velocity and pressure are applied on all six computational boundaries. The grid size of the wing is maintained at half of the fluid grid size. Figure 2(a) shows the wing kinematics, where the flapping motion leads the pitching motion by a phase of $90^{\circ}$. A grid convergence study was performed where the grid size $(\mathrm{dx})$ was systematically decreased from $0.1 \mathrm{c}$ to $0.025 \mathrm{c}$. Figure 2 (b) shows the comparison of lift coefficient $C_{L}$ in three different grids densities. The variation in $C_{L}$ was consistent across the last two consecutive cycles for all three grid sizes, indicating that the flow field had reached a periodic state. The converged solution for $C_{L}$ produced by $d x=0.025 c$ agrees well with the computational result of Dai et al. (2012).

\subsection{A rigid fruit fly plate with passive pitching in hovering flight}

A fruit fly wing in hovering flight with passive pitching is considered to further validate the current solver. Figure 3 shows the shape and dimensions of the wing. The passive pitching mechanism is numerically modelled 
Q. Huang et al., CFD solver validations for simulating passively pitching tandem wings in hovering flight

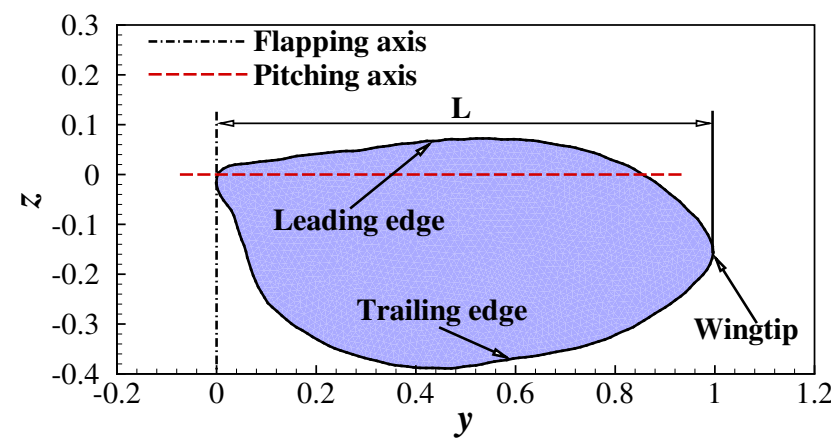

Figure 3. Shape and dimensions of the fruit fly wing model.

Table 2. Fluid domain size in $x-, y-$, and $z$ - directions, finest grid size, grid number, and CPU time $t_{w}$ of one stroke cycle for the computation of the fruit fly wing model. $h$ : hour.

\begin{tabular}{lllll}
\hline Sources & Domain size & Finest grid size & Grid number $\left(10^{6}\right)$ & $t_{w}(h) /$ cycle \\
\hline Lei \& Li (2020) & {$[30 \mathrm{c}, 30 \mathrm{c}, 30 \mathrm{c}]$} & $0.03 \mathrm{c}$ & 6.99 & - \\
Present IB-LBM & {$[$ 30c, 30c, 30c] } & $0.03 \mathrm{c}$ & 12.8 & 2.11 \\
\hline
\end{tabular}

by a torsional spring. The wing has a mean chord length $c$ with an aspect ratio $A R=L^{2} / A=3.2$, where $L$ is the wingspan and $A$ is the wing surface area. The kinematics of the wing are defined as a combination of the prescribed rotation around $z$-axis and the passive pitching motion around $y$-axis.

$$
\phi(t)=-\frac{A_{\phi}}{2} \cos (2 \pi f t)
$$

where $A_{\phi}=7 \pi / 9$ is the flapping amplitude and $f$ is the flapping frequency. In the numerical simulation, the pitching angle $\theta$ is determined by solving the equation of passive feathering motion of the wing, as described in (Kolomenskiy et al. 2019),

$$
J_{y y} \ddot{\theta}(t)+C \dot{\theta}(t)+K_{s}\left(\theta(t)-\theta_{0}\right)=M_{\text {aero }}+J_{z y} \ddot{\phi}(t) \cos \theta(t)+\frac{1}{2} J_{y y} \dot{\phi}(t)^{2} \sin 2 \theta(t),
$$

where $J_{y y}$ and $J_{z y}$ are the moment of inertia, $C=0$ is the damping coefficient of the spring, $K_{s}$ is the torsional stiffness of the spring, $\theta_{0}=0$ is the rest angle and $M_{\text {aero }}$ is the aerodynamic pitching moment on the wing. The non-dimensional groups of the problem include the Reynolds number, mass ratio and the Cauchy number (a ratio between the aerodynamic force and the elastic spring force), which are respectively given by

$$
R e=\frac{U L}{\nu}=300, \quad M=\frac{\rho_{w} h}{\rho c}=1, \quad C h=\frac{\rho A_{\phi}^{2} f^{2} c^{3} L^{2}}{K_{s}}=0.15
$$

where $U=2 f A_{\phi} L$ is the mean wingtip velocity, $\rho_{w}$ is the density of the wing and $h=0.03 c$ is the wing thickness. Table 2 shows the rectangular computational domain that has a size of $30 c \times 30 c \times 30 c$, same as that used in Lei \& $\mathrm{Li}$ (2020). The finest grid around the wing is $0.03 c$, and the total grid number is $12.8 \times 10^{6}$. Six stroke cycles were simulated to ensure that the flow field reached a periodic state. Figure 4(a) shows the time history of the pitching angle, which agrees well with the computational result of Lei \& Li (2020). The grid refinement study here shows that the solutions are converged.

\section{Passively pitching tandem Wings}

Here hovering flight of tandem rectangular wings with passive pitching is simulated. Figure 5 shows the geometric parameters of the wings that have an aspect ratio of $A R=L / c=3.0$. The flapping motions are 


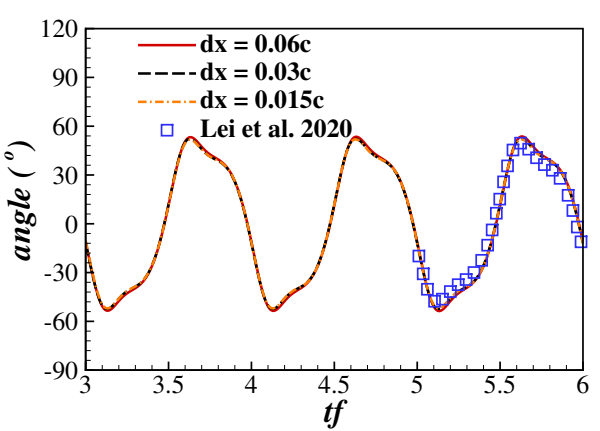

(a) Wing kinematics

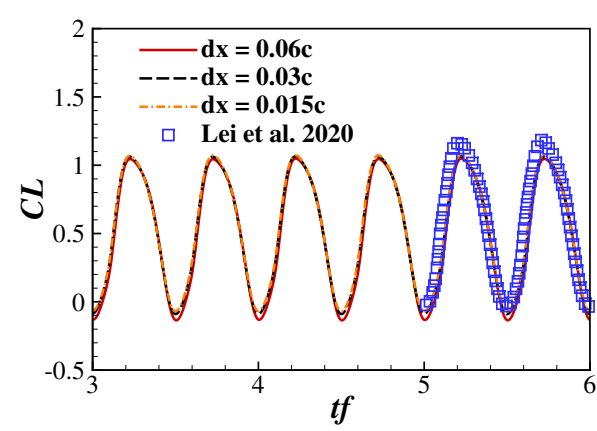

(b) $C_{L}$

Figure 4. Fruit fly wing: wing kinematics and the time history of the lift coefficient in three different grid sizes.

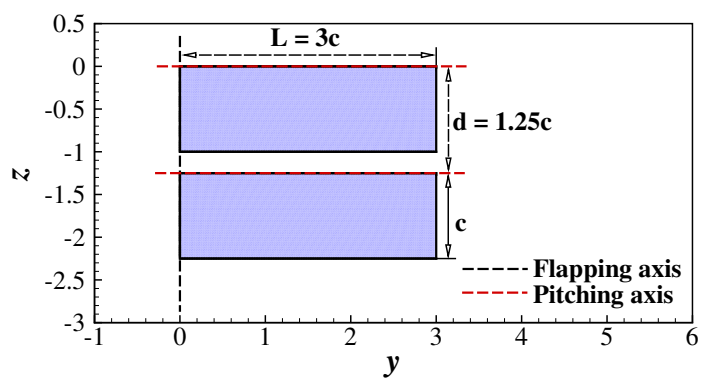

Figure 5. Geometric parameters of the tandem rectangular wings.

prescribed as

$$
\text { Forewing : } \quad \phi_{f}=\frac{\phi_{f 0}}{2} \cos (2 \pi f t) \quad \text { Hindwing }: \quad \phi_{h}=\frac{\phi_{f 0}}{2} \cos (2 \pi f t+\psi)
$$

where $\phi_{f}$ and $\phi_{h}$ are the flapping angle for the forewing and hindwing, respectively. Here $\phi_{f 0}=4 \pi / 9$ is the flapping amplitude and $\psi=\pi$ is the phase angle between the flapping motion of the wings. The pitching motion is solved by equation 18 where the spring damping $C=0$ and wing thickness $h=0.03 c$. The Reynolds number $R e=U L / \nu=300$, the Cauchy number $C h=0.15$ and mass ratio $M=1$ were assumed for both wings. The distance between fore and hind wing $d=1.25 \mathrm{c}$, which nominally matched that of real dragonflies. A rectangular computational domain of size of $30 c \times 30 c \times 30 c$ was used and the finest grid around the wing was $0.04 c$, and the total grid number was $9.21 \times 10^{6}$. Six stroke cycles were simulated. Figure 6(a) shows the forewing kinematics. The time history of the lift coefficient is shown in figure 6(b). The grid refinement study here shows the results are grid independent.

\section{Conclusions}

Validations of an IB-LBM solver for the computations of the hovering flight of prescribed kinematics for a rectangular flapping plate and a rigid fruit fly wing with passive pitching were performed. Good agreements of results between current computations and published data were observed, suggesting the present CFD solver can accurately compute the flow over flapping wing system. The high-fidelity and efficiency of the solver have also been highlighted through the grid convergence study and comparing the computational time with previously published data. The validated solver was then applied to simulate the passive pitching of tandem rectangular wings. This study provides additional data for benchmarking of CFD solvers in the simulation of passively pitching flapping wings. Detailed parameter investigations of passively pitching flapping wings under solitary and tandem configurations will be conducted in future studies. 


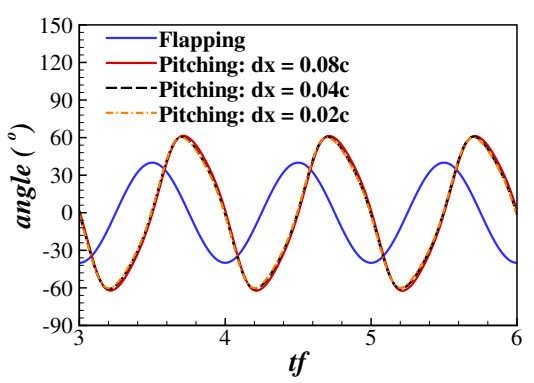

(a) Wing kinematics

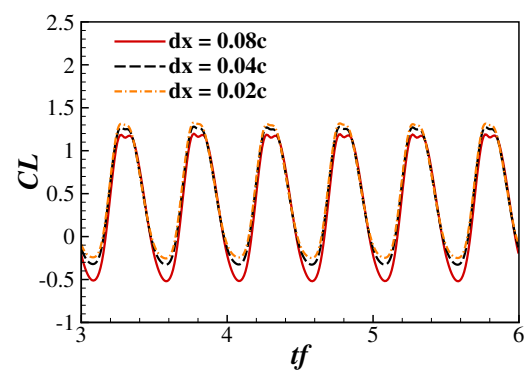

(b) $C_{L}$

Figure 6. Wing kinematics and the time history of the lift coefficient for the forewing in three different grid sizes.

\section{ACKNOWLEDGEMENT}

The authors would like to thank Dr Chengyu Li, Villanova University for discussions on the fruitfly wing simulation. The computation work of this research was performed on the National Computational Infrastructure (NCI) supported by the Australian Government. This work was supported by Asian Office of Aerospace Research and Development (grant nos. FA2386-19-1-4066 and FA2386-20-1-4084) and Office of Naval Research Global (grant nos. N62909-20-1-2088).

\section{REFERENCES}

Dai, H., Luo, H. \& Doyle, J. F. (2012), 'Dynamic pitching of an elastic rectangular wing in hovering motion', Journal of Fluid Mechanics 693, 473-499.

D’Humieres, D., Ginzburg, I., Krafczyk, M., Lallemand, P. \& Luo, L.-S. (2002), 'Multiple-relaxation-time lattice Boltzmann models in three dimensions', The Royal Society 360, 437-451.

Ennos, A. R. (1988), 'The inertial cause of wing rotation in diptera', Journal of experimental biology 140, 161169.

Farrell Helbling, E. \& Wood, R. J. (2018), 'A review of propulsion, power, and control architectures for insectscale flapping-wing vehicles', Applied Mechanics Reviews $\mathbf{7 0 .}$

Guo, Z.-L., Zheng, C.-G. \& Shi, B.-C. (2002), 'Non-equilibrium extrapolation method for velocity and pressure boundary conditions in the lattice Boltzmann method', Chinese physics 11, 366-374.

Huang, Q., Tian, F.-B., Young, J. \& Lai, J. C. S. (2020a), 'A diffused interface immersed boundarylattice Boltzmann method for simulation of channel flow', 22nd Australasian Fluid Mechanics Conference AFMC2020, 7-10 December 2020, Brisbane, Australia .

Huang, Q., Tian, F.-B., Young, J. \& Lai, J. C. S. (2021a), 'Transion to chaos in a two-sided collapsible channel flow', Journal of Fluid Mechanics 926.

Huang, Q., Wang, L., Tian, F.-B., Young, J. \& Lai, J. C. S. (2021b), 'A diffused interface immersed boundarylattice boltzmann method for simulation of stenosis', 14th World Congress on Computational Mechanics \& ECCOMAS Congress 2021, 11-15 January 2021, Paris France .

Kolomenskiy, D., Ravi, S., Xu, R., Ueyama, K., Jakobi, T., Engels, T., Nakata, T., Sesterhenn, J., Schneider, K., Onishi, R. et al. (2019), 'The dynamics of passive feathering rotation in hovering flight of bumblebees', Journal of Fluids and Structures 91, 102628.

Lei, M. \& Li, C. (2020), 'The aerodynamic performance of passive wing pitch in hovering flight', Physics of Fluids 32, 051902.

Lynch, J., Gau, J., Sponberg, S. \& Gravish, N. (2021), 'Dimensional analysis of spring-wing systems reveals performance metrics for resonant flapping-wing flight', Journal of the Royal Society Interface 18, 20200888.

Mazharmanesh, S., Stallard, J., Medina, A., Fisher, A. M., Ando, N., Tian, F., Young, J. \& Ravi, S. (2021), 'Performance of passively pitching flapping wings in the presence of vertical inflows', Bioinspiration \& Biomimetics .

Peskin, C. S. (2002), 'The immersed boundary method', Acta Numerica 11, 479-517.

Yu, D., Mei, R. \& Shyy, W. (2002), 'A multi-block lattice boltzmann method for viscous fluid flows', International journal for numerical methods in fluids 39, 99-120. 